\title{
Predicted CALET Measurements of Heavy and Ultra-Heavy Cosmic Ray Nuclei
}

\author{
B.F. Rauch for the CALET Collaboration* ${ }^{*}$ \\ Department of Physics and McDonnell Center for the Space Sciences, Washington University, \\ St. Louis MO 63130 USA \\ E-mail: brauchephysics.wust1.edu
}

\begin{abstract}
The CALorimetric Electron Telescope (CALET) is a Japanese-Italian-US astroparticle observatory expected to be installed on the ISS in 2015. The main calorimeter (CAL) on CALET is comprised from top to bottom of a charge detector (CHD) with two crossed layers of scintillator paddles, an imaging calorimeter (IMC) with planes of scintillating fibers interleaved with tungsten sheets, and a total absorption calorimeter (TASC) made of lead tungstate logs. The main science objectives of CAL are to measure the combined cosmic ray electron and positron spectrum to 20 $\mathrm{TeV}$, gamma rays to $10 \mathrm{TeV}$, and nuclei $1 \leq Z \leq 40$ to $1,000 \mathrm{TeV}$. In this paper we present the expected numbers of heavy ( $26 \leq Z<30)$ and ultra-heavy (UH) $(30 \leq Z \leq 40)$ Cosmic Ray (CR) nuclei that $\mathrm{CAL}$ will measure in a planned 5 year mission in the full detector geometry accounting for geomagnetic screening and interactions in the CHD. We will also present the numbers of UH CR nuclei that it will measure using the expanded acceptance permitted utilizing the earth's geomagnetic field to screen for events above $\sim 600 \mathrm{MeV} /$ nucleon. Above this threshold the UH charges can be resolved using the CHD with a trajectory correction from the top half of the IMC without the need for energy measurement in the TASC.
\end{abstract}

The 34th International Cosmic Ray Conference,

30 July- 6 August, 2015

The Hague, The Netherlands

\footnotetext{
*Presenter

${ }^{\dagger}$ CALET supported by JAXA in Japan, ASI in Italy and in the USA by NASA grant \#NNX11AE01G.
} 


\section{Introduction}

The CALorimetric Electron Telescope (CALET) consists of the main calorimeter telescope (CAL) and CALET Gamma-ray Burst Monitor (CGBM) subsystems, as shown in the diagram on the left in Fig. . A A side-view of the CAL is shown on the right in Fig. I, which from top to bottom consists of the charge detector module (CHD), imaging calorimeter (IMC), and a total absorption calorimeter (TASC). The CHD is composed of two crossed layers of $32 \mathrm{~mm}$ wide $\times 10 \mathrm{~mm}$ thick $\times$ $450 \mathrm{~mm}$ long EJ200 scintillator paddles. The IMC is $156.5 \mathrm{~mm}$ tall, and is composed of eight $\mathrm{x}-\mathrm{y}$ planes of $4481 \mathrm{~mm}^{2}$ scintillating fibers interleaved with 5 plates of 0.2 radiation length $\left(X_{\circ}\right)$ thick and 2 plates of $1 X_{\circ}$ thick tungsten (total of $3 X_{\circ}$ ) spaced with structural honeycomb. The TASC consists of twelve crossed layers of 16 PWO logs, each $19 \mathrm{~mm}$ wide $\times 20 \mathrm{~mm}$ tall $\times 326 \mathrm{~mm}$ long, for $27 X_{\circ}$. The great depth of the CAL $\left(30 X_{\circ}\right)$ will allow it to measure the electron energy spectrum from $1 \mathrm{GeV}$ to $20 \mathrm{TeV}$, gamma-rays with energies between $10 \mathrm{GeV}$ and $10 \mathrm{TeV}$ and nuclei to 1,000 $\mathrm{TeV}$. The instrument and its main scientific objectives are described in more detail in [四].
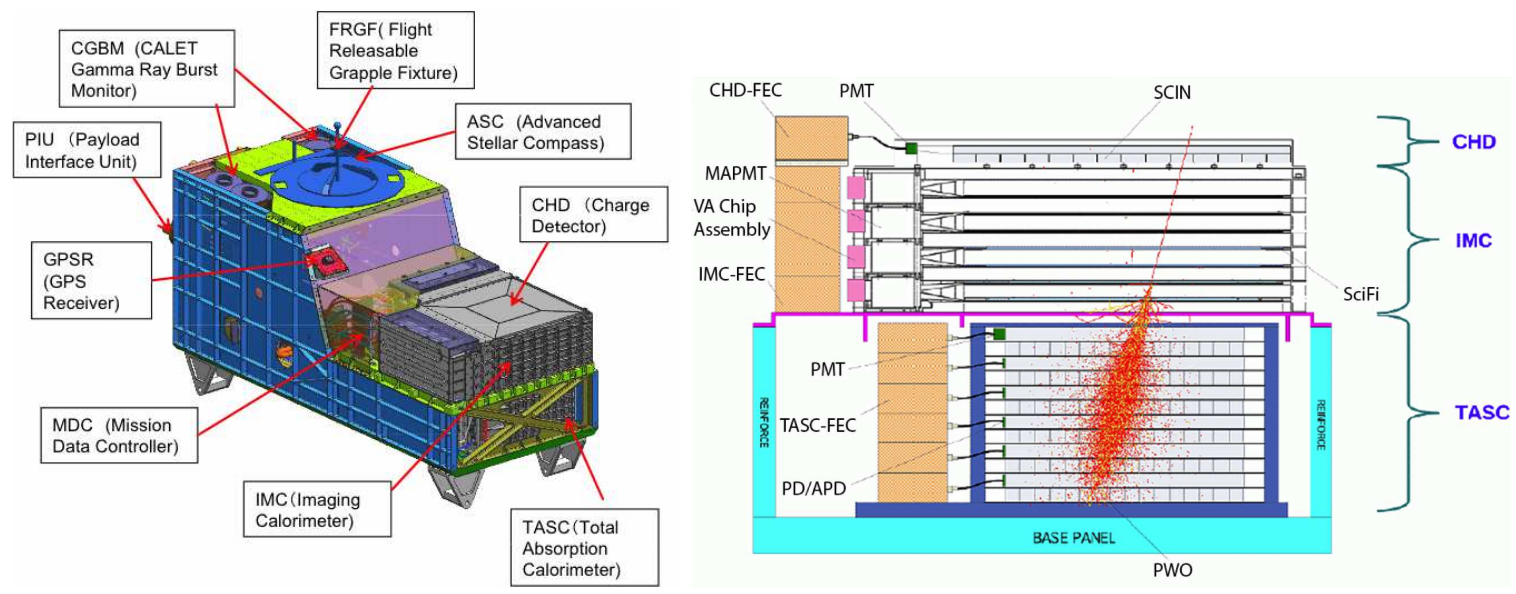

Figure 1: Left: CALET instrument package showing CAL and CGBM subsystems. Right: CAL side-view showing CHD, IMC, and TASC detectors.

\section{Heavy and Ultra-Heavy Galactic Cosmic Rays}

The CHD has the charge resolution [ [ $]$ ] and dynamic range needed to measure Galactic cosmicray (GCR) nuclei up to charge $(Z)=40$, and will also enable the CAL to measure the energy spectra of the more abundant nuclei with $Z \leq 28$ together with the track and energy reconstruction from the IMC and TASC. The rarer ultra-heavy (UH) nuclei $(30 \leq Z \leq 40)$ will also be measured in the CAL, but the statistics collected requiring full passage through the CAL will be limited as the active area decreases from $0.45 \mathrm{~m}$ on a side at the CHD and IMC to $0.32 \mathrm{~m}$ on a side in the TASC, yielding a total geometry factor of $0.07 \mathrm{~m}^{2} \mathrm{sr}$. Fig. $\square$ of GCR relative abundances at $2 \mathrm{GeV} / \mathrm{nuc}$ compared with $\mathrm{SS}$ relative abundances $\left({ }_{14} \mathrm{Si}=1\right)$ for $1 \leq Z \leq 40$ shows that the UH GCR are about $10^{5}$ less abundant than ${ }_{26} \mathrm{Fe}$. In this paper we show the expected cosmic ray nuclei measurement in the charge range $5 \leq Z \leq 40$ for the full instrument geometry, and the UH measurement that can be made by requiring passage through only the $\mathrm{CHD}$ and top IMC layers that yields a larger geometry factor of $0.44 \mathrm{~m}^{2} \mathrm{sr}$, which was first shown in [B] and refined in [䧃] and [可]. 


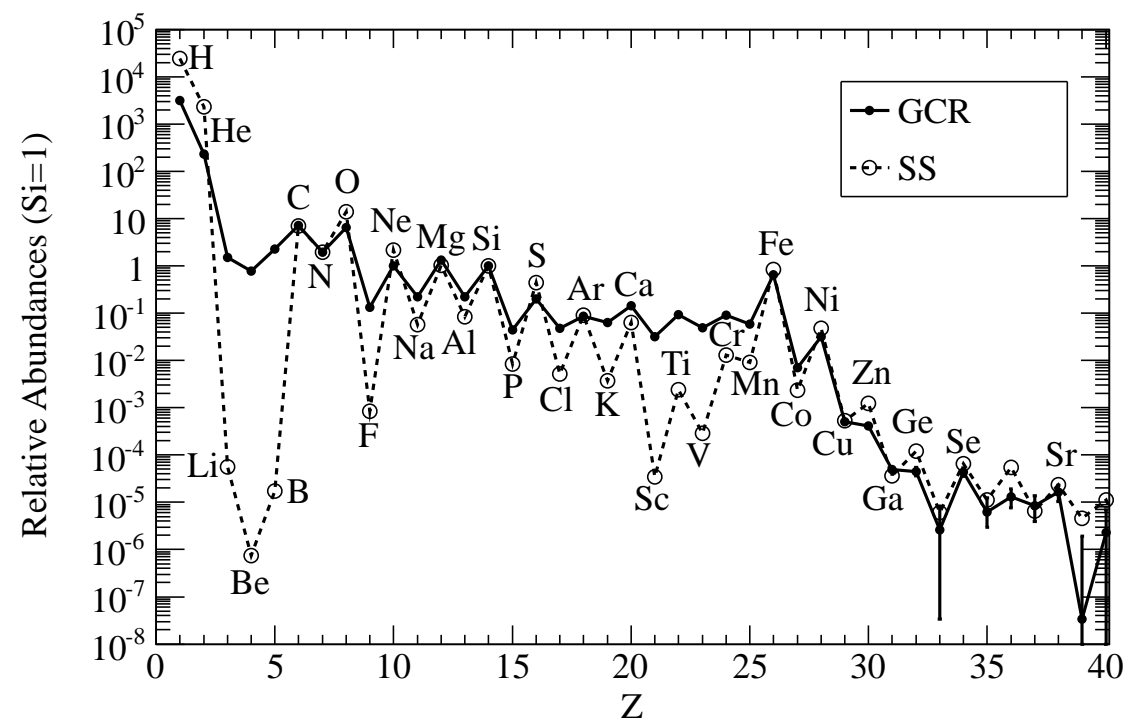

Figure 2: Solar System (SS) relative abundances [6] and Galactic cosmic-rays (GCR) relative abundances

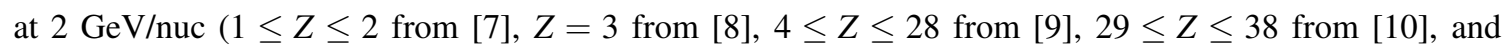
$39 \leq Z \leq 40$ from [प]].) normalized to $\mathrm{Si}=1$.

\section{Charge Identification Using Energy Threshold Cut}

In order to maximize CALET's measurement of UH nuclei it is necessary to optimize its geometric acceptance of them, which can be achieved if the UH charges can be determined without an energy correction from the TASC. The two layers of $1 \mathrm{~cm}$ thick scintillator in the CALET CHD are similar to the two $0.8 \mathrm{~cm}$ thick scintillators at the top of the TIGER instrument [प0]], so the signal dependence of the TIGER scintillators in the UH region is expected to be representative of those used in CALET. The CHD scintillator (EJ200) has a saturated response proportional to that of EJ204 $S \sim Z^{1.61}\left[[]\right.$ ], and the TIGER Saint-Gobain BC-416 scintillator has a response $S \sim Z^{1.69}$ [Ш]]. Accelerator studies of scintillator response have shown similar scintillator saturation results for nuclei up to ${ }_{47} \mathrm{Ag}$ [ए2] , which indicates that the response will be the same for the UH GCR.

The left plot in Fig. B shows the dependence of the combined signals of the top two TIGER scintillators $(\mathrm{S} 1+\mathrm{S} 2)$ as a function of acrylic Cherenkov signal $(\mathrm{C} 1)$ for cosmic ray nuclei on the 2003 TIGER flight [四]. The charge contours show that the scintillator signal decreases with increasing energy to minimum ionization and then increases at the relativistic rise and quickly saturates. Taking events with energies above the threshold line shown selects from regions of the charge contours with comparatively small signal dependence on energy. The right plot in Fig. [3] shows histograms of summed scintillator $(\mathrm{S} 1+\mathrm{S} 2)$ signals for 2003 TIGER ${ }_{26} \mathrm{Fe}$ events for all energies (black), and for all energies above the threshold (red: $\mathrm{C} 1>5000, \sim 600 \mathrm{MeV} /$ nucleon), which yields a nearly Gaussian distribution for the charge peak. This shows that above the $\sim 600$ $\mathrm{MeV} /$ nucleon threshold charges can be determined without an energy correction that would require passage through the TASC, which greatly increases the geometric acceptance from $0.07 \mathrm{~m}^{2} \mathrm{sr}$ to $0.44 \mathrm{~m}^{2} \mathrm{sr}$. 

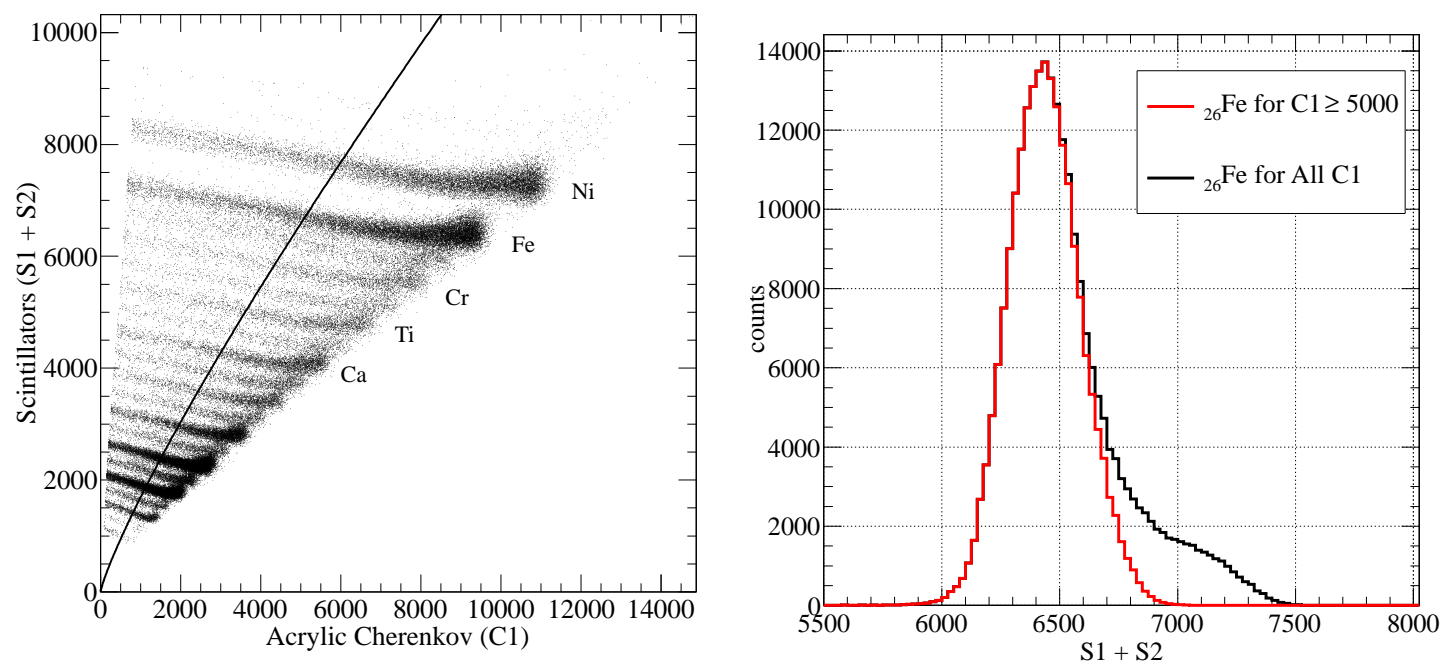

Figure 3: TIGER data from 2003 flight [W]. Left: Scatter plot of the sum of top scintillator signals (S1 + S2) versus acrylic Cherenkov signal (C1). Curve is $600 \mathrm{MeV}$ /nucleon threshold. Right: Sum of top scintillator signals $(\mathrm{S} 1+\mathrm{S} 2)$ for ${ }_{26} \mathrm{Fe}$ events for all energies (black line) and for acrylic Cherenkov signal $\mathrm{C} 1>5000$ ( $600 \mathrm{MeV} /$ nucleon) (red line).

\section{Rigidity Cuts for ISS Orbit}

The earth's magnetic field can be used to provide the energy threshold cut described in the

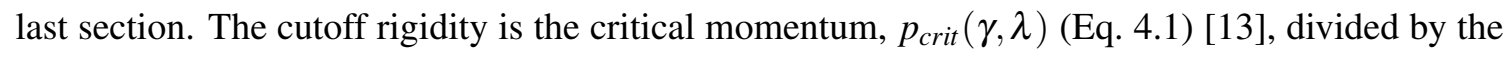
atomic number, $R_{\text {cutoff }}=p_{\text {crit }}(\gamma, \lambda) / Z$, which is shown in the left plot of Fig. 困 as a function of geomagnetic latitude $(\lambda)$ and the transverse East-West angle relative to the field lines $(\gamma)$. Eq. 4.2 shows that the energy threshold $E_{c r i t}$ depends on the ratio of critical momentum to atomic mass, and so (at a fixed cutoff rigidity) by the ratio of atomic number to atomic mass. This means that UH nuclei are screened less effectively than lower charge nuclei, providing a slight positive bias in their acceptance on the ISS. It also means that in the UH charge identification method the critical momentum for the threshold energy will be proportionally higher for UH nuclei.

$$
\begin{gathered}
p_{\text {crit }}(\gamma, \lambda)=60 Z\left[\frac{1-\sqrt{1-\cos (\gamma) \cos ^{3}(\lambda)}}{\cos (\gamma) \cos (\lambda)}\right]^{2} \\
E_{c r i t}=\sqrt{p_{c r i t}^{2} / A^{2}+m_{a m u}^{2}}-m_{a m u}
\end{gathered}
$$

The geomagnetic latitudes for $p_{\text {crit }}(\gamma, \lambda)$ seen in the ISS orbit shown on the right in Fig. 团 have been derived from the geomagnetic vertical cutoff rigidities at an altitude of $450 \mathrm{~km}$ [[1]] using $R_{\text {cut }}=15 \cos ^{4}(\lambda) \mathrm{GV}$ [ए3] after interpolating the vertical cutoff rigidities to a $1^{\circ}$ pitch. The magnetic rigidities at $450 \mathrm{~km}$ are expected to be representative of those CALET will see at $\sim 400$ $\mathrm{km}$ on the ISS $51.6^{\circ}$ inclination orbit shown by the black curves in the plot on the right in Fig. $\mathbb{\text { t. }}$ The region of the South Atlantic Anomaly (SAA) is shown in white, and has been derived from the region where the AMS-02 instrument already on the ISS cannot take data [L5]. The SAA mask 

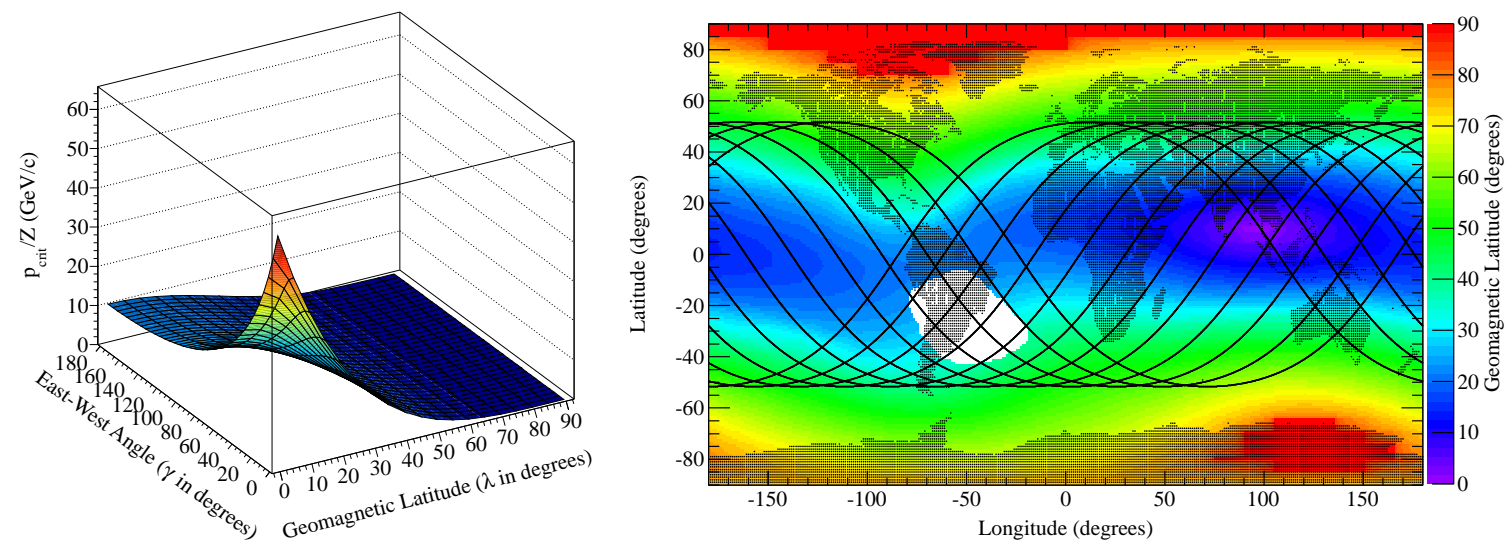

Figure 4: Left: Cutoff rigidity (critical momentum per charge). Right: Contour plot of geomagnetic latitude at $450 \mathrm{~km}$ in $1^{\circ}$ longitude and latitude bins derived from [ㅍ]. ISS orbit of $51.6^{\circ}$ inclination is shown in black curves and SAA shown in white.
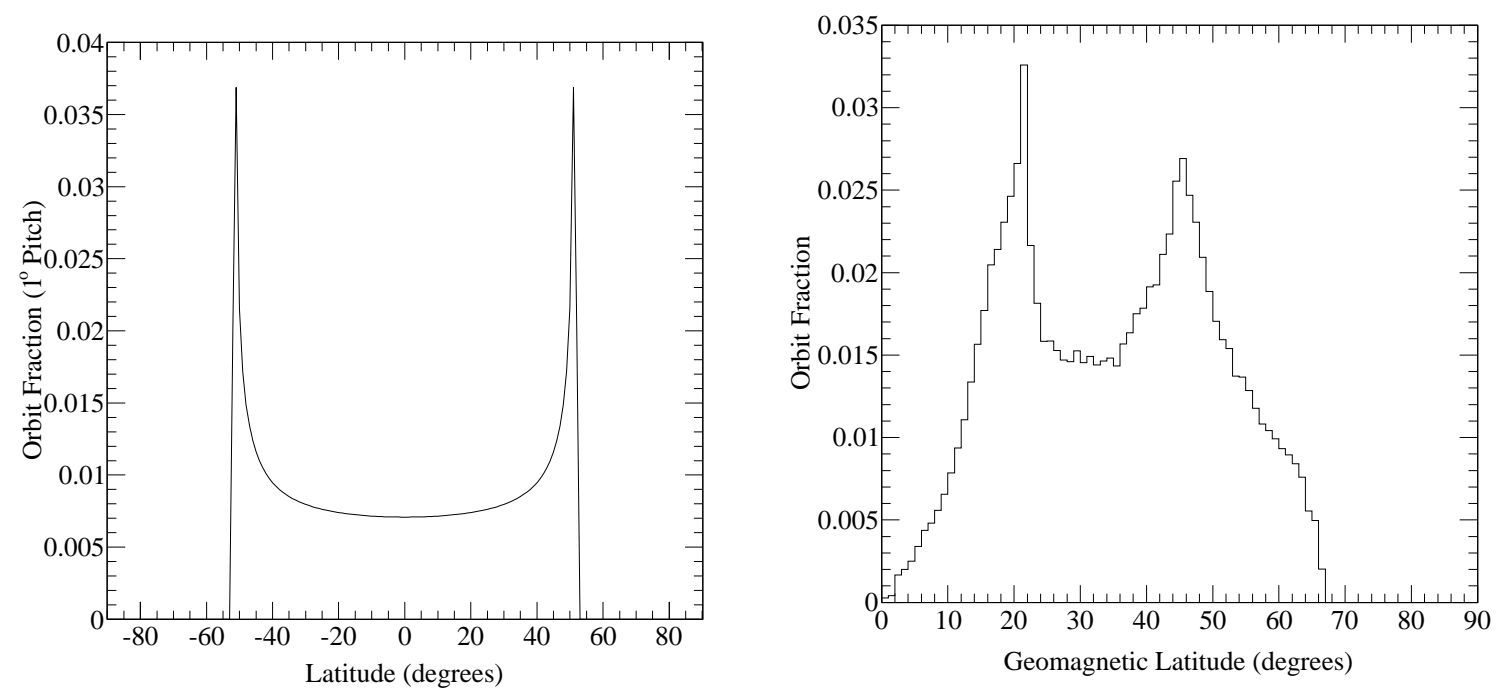

Figure 5: Left: ISS orbit fraction as a function of geographic latitude. Right: CALET operational geomagnetic latitude residence time.

has been applied as a conservative estimate and represents a reduction of $\sim 6 \%$ of the observation time; however, it is expected that CALET will be able to observe heavy nuclei in this region with its high energy trigger. The geographic latitude orbit fraction shown in the left plot of Fig. 1 is then used to determine the geomagnetic latitude orbit fraction shown in the right plot of Fig. [1.

\section{Predicted CALET Observations}

The GCR spectra are required to predict the numbers of nuclei that CALET will measure, which requires estimating the as yet unmeasured UH spectra. CALET is expected to see intermediate Solar modulation during its mission, so integral spectra derived from averaged 

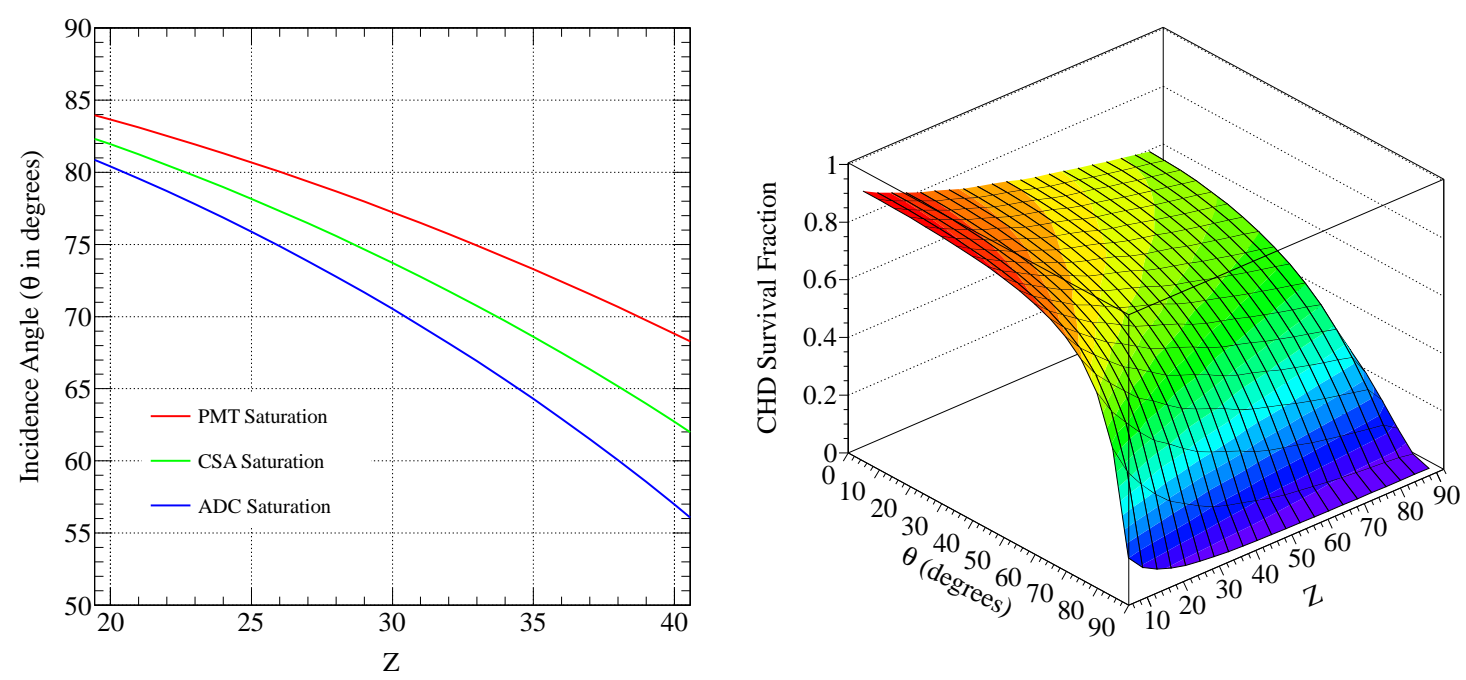

Figure 6: Left: CHD saturation curves as a function of zenith angle $\theta$ and charge $Z$ for the CHD electronics chain. Right: Fraction of events passing through CHD without interacting.
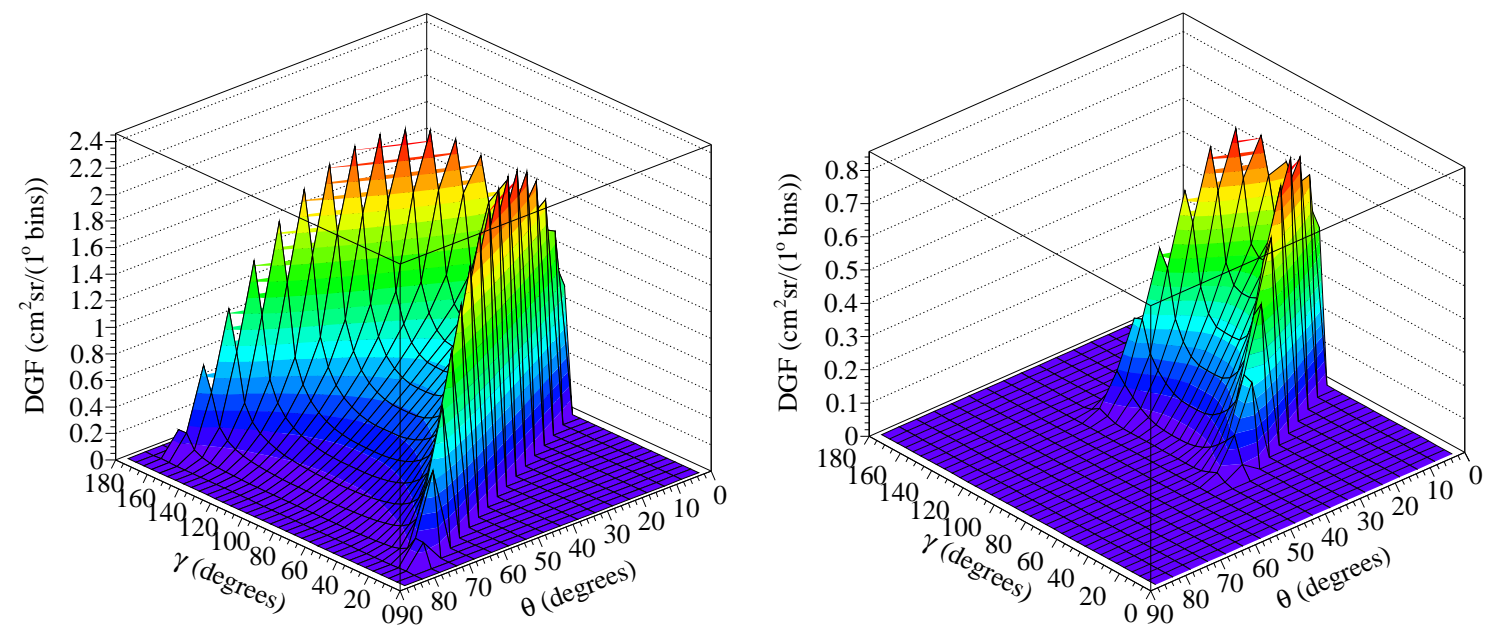

Figure 7: Differential geometry factors as a function of East-West angle $(\gamma)$ and zenith angle $(\theta)$. Left: for UH mode requiring passage through CHD and top 4 IMC layers. Right: for full instrument geometry.

differential spectra in [ए]] for Solar minimum and maximum are used for $5 \leq Z \leq 28$. The UH GCR are mostly primary in composition, so their spectra are derived by scaling the integral ${ }_{26} \mathrm{Fe}$ spectrum with relative abundances: HEAO-3-C2 for $26 \leq Z \leq 29$ [Q] and TIGER for $30 \leq Z \leq 40$ [एँ]].

For the UH mode the abundances that CALET will see for each element are estimated utilizing their integral spectra to find the events expected at each $1^{\circ}$ geomagnetic latitude $(\lambda)$ step as a function of each $1^{\circ}$ East-West angle $(\gamma)$ step. At each $\gamma$ where the critical momentum corresponds to a kinetic energy ( $E_{\text {crit }}$ Eq. 4.2$)$ greater than the $600 \mathrm{MeV} /$ nucleon threshold the integral spectrum 
is evaluated at $E_{\text {crit }}$ and multiplied by the fraction that does not interact in the CHD if the detector does not saturate at that incidence angle. CHD saturation in the electronics chain is limited by the analog to digital converters (ADC), and is shown as a function of charge and incidence angle in the left plot in Fig. 6. The interaction survival probability in the CHD as a function of charge and incidence angle is shown in the right plot of Fig. 6 . The fluxes at each of these bins are multiplied by the differential geometry factor for the UH-trigger, which includes the CALET CHD and top

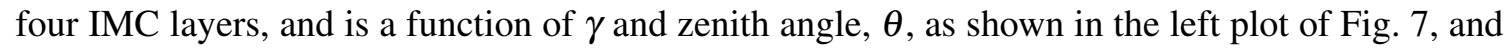
by the expected 5 year exposure time on the ISS. For the full geometry acceptance the method is similar, with the differences being that there is no threshold cut on $E_{c r i t}$ and the smaller differential geometry factor for the full instrument shown in the right plot of Fig. $\square$ is used.
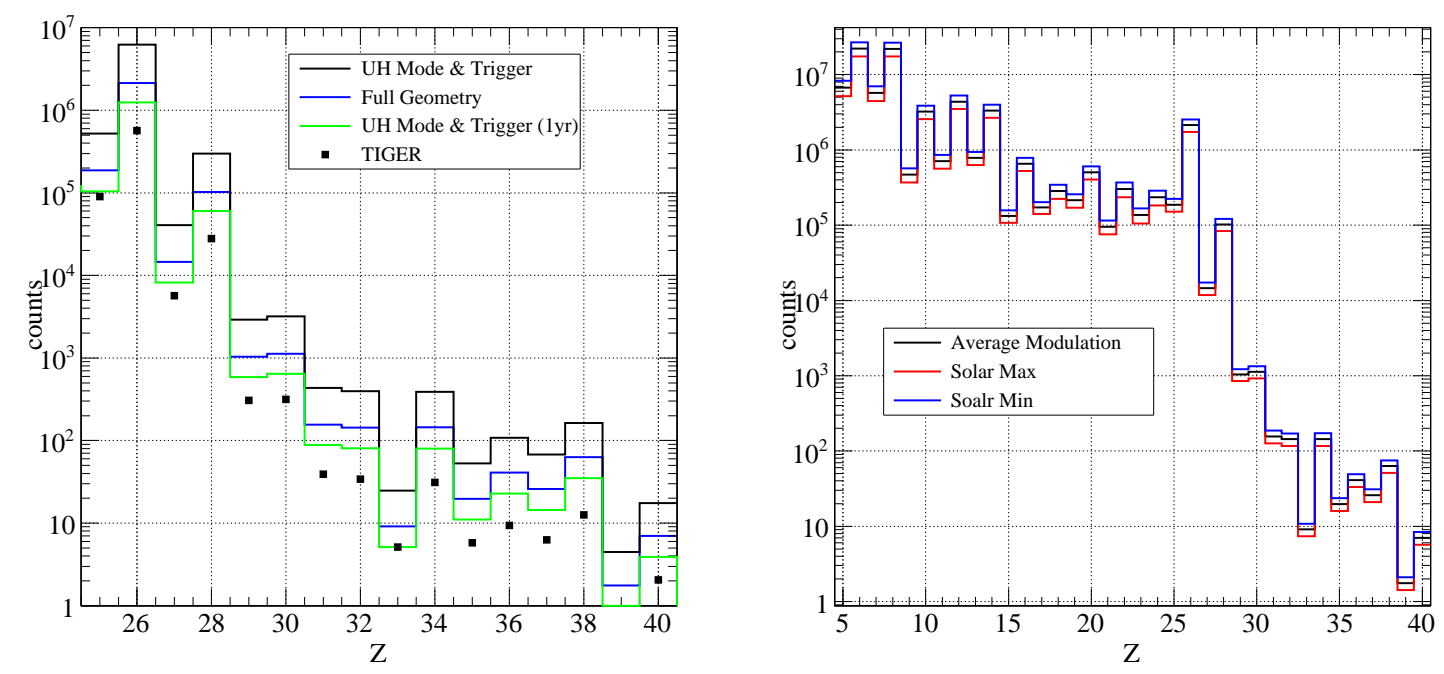

Figure 8: Left: Predicted CALET UH GCR observations. Right: Predicted CALET heavy and UH GCR for full instrument geometry.

\section{Conclusions}

The left plot in Fig. 8 shows the expected CALET UH event numbers over an anticipated 5 year mission compared with those measured by TIGER (points) [ए]] at balloon altitudes for the UH (black) and full geometry (blue) measurement modes, and the right plot shows the expected event numbers for the full acceptance over $5 \leq Z \leq 40$ for intermediate (black), maximum (red) and minimum (blue) solar modulations. CALET is expected to measure $\sim 10 \times$ the UH events as TIGER with the UH mode, and $\sim 4 \times$ the UH statistics with the full geometry and energy reconstruction. Previously reported predicted CALET UH measurements were calculated for one year only, which are shown in the green histogram on the left in Fig. \&. The elements where the CALET one year measurement is approximately the same as TIGER (25 $\mathrm{Mn}$ and $\left.{ }_{33} \mathrm{As}\right)$ are enhanced at TIGER by atmospheric interactions of more abundant higher- $Z$ elements. The CALET measurements will be free of systematic effects resulting from atmospheric propagation corrections 
and will complement the higher statistics balloon-borne SuperTIGER measurements in the $30 \leq$ $Z \leq 40$ range.

\section{References}

[1] S. Torii for the CALET Collaboration, Calorimetric Electron Telescope (CALET) for the ISS, in proceedings of The 34th International Cosmic Ray Conference, POS (ICRC2015) 581 (2015).

[2] P.S. Marrocchesi et al., Beam test performance of a scintillator-based detector for the charge identification of relativistic ions, NIMPA, 659 (2011) 477-483.

[3] B.F Rauch et al., Capability of the CALET Experiment for Measuring Elemental Abundances of Galactic Cosmic Ray Nuclei Heavier than Nickel $(Z=28)$, in proceedings of The 32nd International Cosmic Ray Conference, 6, 348-352 (2011).

[4] B.F. Rauch for the CALET Collaboration, CALET Measurement of Ultra-Heavy Cosmic Rays, in proceedings of The 33rd International Cosmic Ray Conference, paper id 0819 (2013).

[5] B.F. Rauch for the CALET Collaboration, Predicted CALET measurements of ultra-heavy cosmic ray relative abundances, AdSpR, 53, 10 (2014) 1444-1450.

[6] K. Lodders, Solar System Abundances and Condensation Temperatures of the Elements, ApJ, 591 (2003) 1220-1247.

[7] T. Sanuki et al., Precise Measurement of Cosmic-Ray Proton and Helium Spectra with the BESS Spectrometer, ApJ, 545 (2000) 1135-1142, [arXiv: astro-ph / 0002481 ].

[8] M. Aguilar et al., Isotopic Composition of Light Nuclei in Cosmic Rays: Results from AMS-01, ApJ, 736 (2011) 105-116, [arXiv:1106.2269].

[9] J.J. Engelmann et al., Charge composition and energy spectra of cosmic-ray nuclei for elements from Be to NI - Results from HEAO-3-C2, A\&A, 233 (1990) 96-111.

[10] B.F. Rauch et al., Cosmic Ray origin in OB Associations and Preferential Acceleration of Refractory Elements: Evidence from Abundances of Elements ${ }_{26}$ Fe through ${ }_{34} \mathrm{Se}, \mathrm{ApJ}, 697$ (2009) 2083-2088, [arXiv:0906.2021].

[11] B.F. Rauch, Measurement of the relative abundances of the ultra-heavy galactic cosmic rays $(30<=$ $Z<=40$ ) with the Trans-Iron Galactic Element Recorder (TIGER) instrument, Ph.D. thesis, Washington University, St. Louis 2008.

[12] W.R. Binns et al., Response of Scintillators to UH Nuclei, in proceedings of The 22nd International Cosmic Ray Conference, 2, 511-514 (1991).

[13] C. Störmer, The Polar Aurora, Oxford University, London 1950.

[14] D.F. Smart \& M.A. Shea, Fifty years of progress in geomagnetic cutoff rigidity determinations, AdSpR, 44 (2009) 1107-1123.

[15] S. Schael for the AMS Collaboration, Precision measurements of the electron spectrum and the positron spectrum with AMS, in proceedings of The 33rd International Cosmic Ray Conference, paper id 1257 (2013).

[16] J.S. George et al., Elemental Composition and Energy Spectra of Galactic Cosmic Rays During Solar Cycle 23, ApJ, 698 (2009) 1666-1681.

[17] B.S. Nilsen et al., Fragmentation cross sections of relativistic ${ }_{36}^{84} \mathrm{Kr}$ and ${ }_{47}^{109} \mathrm{Ag}$ nuclei in targets from hydrogen to lead, PhRvC, 52 (1995) 3277-3290. 